\title{
Relevance of it in libraries: Desire a tread a front to advanced library in current trends
}

\author{
Ranjeet Pagore Bhanudas
}

Librarian, MSP Mandal's R. B. Attal College, Georai Dist. Beed, Maharashtra, India

*Corresponding Author: Pagore R.B

Email: pranjeet23@gmail.com

\begin{abstract}
Smart is a network which allows free access to all digital libraries in the electronic society. Smart libraries is a cyber-security and well-being behavior change program designed to equip libraries and link users of libraries with the skills they need to use technology in a smart, healthy and responsible manner. Being smart means knowing how to protect against online security and privacy threats, downloading content in a legal and ethical way, researching and referencing information, and managing cyberspace reputations and relationships. Smart helps to incorporate a culture of constructive use of technology, establish policies, processes, and gain access to evidence-informed resources and track progress in being intelligent. The term internet is a major component of daily life, and libraries are the information centers that are at the forefront of delivering the resources required to access and support the community. Smart enables libraries to provide a good atmosphere in which people can experience feelings of security, become part of real and virtual communities, and learn the skills they need. We believe that community-based acts such as smart libraries can really push e-safety worldwide as a cultural standard in communities.
\end{abstract}

Keywords: Advanced Library, Solid Communities, economic wellbeing, Cloud computing, Web 2.0, E-Granthalaya 4.0, Freeware Applications, etc.

\section{Introduction}

The Web is now changing so rapidly that it is nothing but a library solution in a digital way to determine if anything is really new. It is also a very sophisticated solution for true multimedia streaming and digital distribution through an agnostic computer and browser. The library management system has developed with or without a digital format for more than 20 years. Web-based solutions, the latest smart version based on what all users want and need in a smart or digital library with a focus on esthetics, are pleasing to the eye and easy to use for both library staff and users. It is a free approach to make the library feel secure, integrating cyber security into its policies, agreements, growth of personnel, organizational actions and culture, training, and day-to-day operations. A system to stay on top of cyber-safety problems that enables the library to respond to accidents and negative actions and to promote constructive use of meaning. A way to strengthen library group guidance and training, providing them with the skills they need to use emerging technologies securely and comfortably, get the most out of digital technology, and prevent cyber threats such as scams and cyber bullying. ${ }^{1}$

\section{Basic Functionality}

The advanced library platform is a holistic structure that provides the library community with skills and tools to handle cyber-issues confidently and continue to accept the positive aspects of online operation. Smart libraries provide a free and comprehensive approach by providing a well-structured action plan that connects to best practice tools to help libraries find weaknesses in their policies and operations. Using an online system tool used by library practitioners, the library can track progress and access services.

Smart libraries help library staff develop their direction and preparation for library users by connecting the structure, actions and services, giving libraries what they need. The interactive tool. To get the most out of digital technology and prevent cyber-risks, consumers learn the skills they need to use digital technologies comfortably and safely. The most important cyber-safety services and realistic tools are put together in one place. Smart libraries are designed to be adaptable and versatile to all forms of libraries, including academic, public, metropolitan, regional, rural, remote and mobile libraries, offering valuable guidance to libraries that may have taken some treads to raise knowledge and skills in cyber security, digital literacy and digital citizenship, as well as those who are keen to get started. ${ }^{2}$

The introduction of intelligent libraries helps minimize hazards. Libraries will show their success in incorporating cyber-safety and health into their library activities by engaging in the program. By encouraging workers and consumers to increase their awareness and skills to navigate the online environment in a secure, intelligent and responsible manner, smart technology helps to minimize the digital divide. Smart libraries for the latest scenario or the digital era are an extension of an established model.

\section{Consumed Technology for Advanced Library}

The majority of libraries contribute to digital citizenship in a smart way. It should be the first place to introduce the most innovative technologies. Libraries today are not just about borrowing books. These are imaginative spaces, not just for people, but also communities. These are incubators and learning centers for the economy. Above all, libraries are points of entry to the modern world. These are the means by which technology is applied and digital exclusion is avoided. Consequently, libraries should be fitted with sufficient updating technology in order to boost the technical literacy in local communities. The following technologies are also useful in changing or converting a standard digital library into a smart one. ${ }^{3}$

1. Library bookmark and influence 
2. Augmented actuality app

3. Book delivery buzz

4. Digital network for print books

5. Library Gadget

6. Mobile library center

7. Print on demand machines

8. Access to library via simply used app

9. Library direction technology

10. Wi-Fi / Internet passage with $3 \mathrm{G} / 4 \mathrm{G}$ speed network

\section{Benefits}

\section{Solid Communities:}

Libraries may be key partners in solving the challenges of social exclusion, vulnerabilities, fragmented societies and ill health. Libraries can connect communities and transform lives. It offers safe spaces in the center of their neighborhoods and also provides many services to people living on a low poverty line, lonely, unemployed or elderly, and people with medical conditions or disabilities in the long term. ${ }^{4}$

Mobile libraries that are smart can help combat rural alienation and social exclusion. Smart libraries can provide insurance and health care information as well as prescription books. Smart libraries will help strong, integrated communities and provide access to public domain information on local and national issues.

1. Healthy and healthier neighborhoods

2. Thriving quarters

3. A deep mutual culture

4. Tackling poverty and alienation from society

5. Longer, more healthy lives

6. Reduced health gaps

7. Addressing drawbacks

\section{Behave as cultural centre}

Smart libraries, through e-books and e-literature, music, film and theatre, will encourage people. Libraries are places where individuals can be innovative, where groups can interact through modern technologies to explore a common interest in the area of arts and crafts through cultural events. ${ }^{5}$

1. Enriching the lives of citizens and communities

2. Placing art and community at the center of regeneration

3. Promoting the social and economic position of arts and culture

4. Production of a competitive creative economy

\section{Well supporting learning}

Smart libraries encourage learning at all stages of life, providing resources for everyone in modern society, from children to the elderly. Advanced library staff can help individuals understand and act on data that can make a significant difference to their well-being or emerging technologies.

1. Creating possibilities

2. Addressing drawbacks

3. Creating cleverer people

4. Helping all to fulfill their potential

5. Make opportunities fairer

6. Enhancement of skills for jobs

\section{Promoting economic wellbeing}

Smart libraries assist job seekers, identify prospects and plan for the creation of sustainable carriers. They offer courses and training in digital skills that enable individuals to pursue employment and to support the growth of small businesses. It will provide vital assistance in the digital future for individuals applying for welfare benefits. ${ }^{6}$

1. Growth and sustainable employment

2. A richer and fairer world

3. Allowing company to generate employment

4. Development of sustainable economic growth

\section{Impact on Society}

Smart libraries are a type of information technology in which, as well as technical development, social impact matters. New technology in the absence of actual users and large sets is difficult to test. Through conducting multi-year large-scale research projects that develop real-world electronic test beds used by actual users and seeking to develop innovative comprehensive and user-friendly solutions for digital libraries, the best way to develop successful new technologies is to develop them. ${ }^{7}$

The WWW (World Wide Web) has made Internet access part of the system of daily life. Every day, millions of people around the world access the internet. Institutions of higher education, under growing pressure to enhance results, are making well-documented and substantial investments in emerging technologies. Modern society's schools, universities and public users scramble to collect data, assess results, and demonstrate success well. Personalized learning technology is at the core of this transition, helping users distinguish instruction and think differently about the delivery of text for instructional material. Although indexing the library repositories of an organization is significantly more difficult than open web sites, it is now possible to crawl, index, enrich and match thousands of digital assets (e-books, e-serials, audio and video) every minute. ${ }^{7}$

Imagine the effect on access to materials, as the use of buried, digital academic content is growing by over $\$ 1$ billion. But the role of the library in driving this digital transformation does not end with access. First of all, smart libraries have the ability to make Netflix-like content recommendations based on the findings of students, faculty, research scholars or user preferences.

\section{Application of Current Advances Cloud computing}

Cloud computing is a kind of Internet-based computing that provides computers and other devices on demand with shared processing resources and data. Cloud computing and storage systems provide consumers and companies with different capabilities to store and process their data in third-party data centers as a model for allowing ubiquitous, on-demand access to a common pool of configurable computing resources (e.g., networks, servers, storage, software, and services). Due to the benefits of high processing capacity, cheap service costs, high performance, scalability, accessibility as well as 
affordability, cloud computing has become a highly demanded service or utility. ${ }^{8}$

A general concept for the distribution of hosted services over the Internet is cloud computing. The objective of cloud computing is to apply conventional supercomputing or highperformance computing capacity, usually used by military and research facilities, to perform tens of trillions of computations every second, to deliver personalized information, to provide data storage or to power massive, immersive online computer games in consumer-oriented applications such as financial portfolios. Cloud computing services can be classified differently as private, public or hybrid. ${ }^{9}$

\section{Web 2.0}

Describes sites on the World Wide Web that emphasizes material, usability, and interoperability created by users. In comparison to websites where people are restricted to the passive viewing of content, a web 2.0 platform may allow users to connect and collaborate with each other in a social media dialogue as creators of user-generated content in a virtual community. Web 2.0 is a compilation of internet trends and resources for use. In other disciplines, the idea of web 2.0 has arisen and modified the whole mode of practice of library science as well, and a new concept of library 2.0 has been implemented. ${ }^{10}$

\section{E-Granthalaya 4.0}

NIC Library and Information Technology Division's library automation program. The program was developed to take into account the specifications of special libraries and government libraries. The program includes various modules such as Management, Book Acquisition and Cataloguing, Distribution, Monitoring of Serials, Indexing of Posts, Budget Controls, and OPAC etc. ${ }^{11}$

Integrated Library Management Program from the National Informatics Centre (NIC), Department of Electronics \& Information Technology, Ministry of Communications and Information Technology, Government of India, is the digital agenda for library automation and networking. The software has been developed by a team of experts from software as well as Library and Information Science discipline. The software is useful for the automation of library in-house operations and offers various member services online. The app offers an integrated OPAC web interface to publish a catalog of libraries over the Internet.

Thus, the app is compatible with UNICODE and supports data entry in local languages. The new eGranthalaya edition, i.e. Ver.4.0 is a 'Cloud Ready Framework' that offers, in business mode, a web-based data entry solution with a centralized library cluster database. As the back-end database solution, E-Granthalaya 4.0 uses Postgre SQL-an Open Source DBMS. E-Granthalaya 4.0 is made available in the NIC National Cloud for Government Libraries Only on request with free application hosting and online access databases for all smart libraries. ${ }^{11}$

\section{RFID}

The new technology to be used in library theft detection systems is RFID (Radio Frequency Identification). It allows a library book to be monitored and radio waves to interact with it. This technology is similar to a mobile phone in nature. For technologies that use radio waves to automatically classify people or objects, RFID is a broad term.

In library circulation operations and theft detection systems, RFID can be used. RFID-based systems go beyond security to become monitoring systems that combine security with more effective library-wide material tracking, including simpler and faster loading and unloading, inventorying, and handling of materials. ${ }^{12}$

This technology allows librarians, when charging and discharging objects, to minimize valuable staff time spent scanning barcodes. RFID is a mixture of microchip technology and radio-frequency based technology. Information found in the tags attached to library materials on microchips is read using radio frequency technology, regardless of object orientation or proper alignment.

\section{Freeware Applications for Digital Repositories D-Space}

It is software for institutional registry applications. For academic, non-profit, and commercial organizations creating open digital repositories, D-Space is the software of choice. Installing "out of the box" is free and simple and fully customizable to suit every organization's needs. With an ever increasing group of developers, dedicated to constantly developing and enhancing the app, D-Space maintains and allows quick and open access to all forms of digital content, including text, pictures, moving images, mpegs and data sets. $^{13}$

\section{Greenstone}

Is a suite of tools for creating and distributing smart/digital library collections. It offers a modern way of storing and publishing information on the Internet or on CD-ROM. Greenstone is developed and distributed in collaboration with UNESCO and the Human Info NGO by the New Zealand Digital Library Project at the University of Waikato. It is open-source, multilingual software released under the terms of the GNU General Public License. ${ }^{13}$

\section{For associated Library Software (ILS)}

Evergreen, Koha, Open Biblio, New Gen Lib (NGL), SOPAC (Social On-line Public Access Catalogue). ${ }^{14}$

\section{Evergreen}

Highly scalable advanced library software that helps library patrons locate materials from libraries and helps libraries track, index, and circulate those materials. Open source software, freely available under the GNU GPL, is Evergreen.

\section{Koha}

The first automation kit for the free and open source software library (ILS). Creation is funded by libraries from around the world of different styles and sizes, volunteers, and support 
firms. For smart libraries of all sorts, Koha has become a viable, scalable solution. With its advanced features set, Koha is designed on this foundation; Koha is today the most technically advanced open source ILS on the market.

\section{Open Biblio}

It is an open source, easy-to-use, integrated library framework written in PHP that includes OPAC, circulation, cataloging, and functionality for staff administration. The aim of this project is to provide private collections or public libraries with an affordable library automation solution.

\section{New Gen Lib (NGL)}

This is the product of a partnership between the Institute of Information and Knowledge Management of Verus and Kesavan. NGL is developed and maintained by Verus Solutions and Kesavan Institute has provided the domain expertise. It offers several simple ILS functions and has many built-in social media functions.

\section{SOPAC (Social Online Public Access Catalog)}

It is a Drupal CMS module that provides the power of the Drupal content management system with the true integration of your library catalog system while allowing users to tag, rate, and review your holdings. In order for SOPAC to become a genuinely community-driven catalogue system, user feedback is then integrated into the discovery index.

\section{Conclusion}

As a result, through digital technology in various software applications, the advanced library is intended to provide all library resources quicker, better and smarter to its end users. Smart libraries, as social learning and communication environments, present educational and knowledge facilitators with rapid distribution of information in the digital age. Smart libraries emphasize their activities in order to provide the people of modern society with popular knowledge. In other words, an informal, collaborative learning location and process that allows all types of visitors to connect, contribute, participate and engage in a smart way with the advanced library. In a positive sense, this new dynamic and scientific technological process contributes to the collaborative, social construction and sharing of information and knowledge among smart users. Worldwide, intelligent libraries have an immense capacity to promote open and free sharing and human participation in creative open source software design. Users can use shared physical spaces that can build social and spatial barriers, allowing them to participate and share in social learning experiences through smart libraries from very different backgrounds. The Advanced library's vision is to create a "indoor living lab" where students and researchers can build, test and present smart technology, access and analyze the data collected in order to carry out both qualitative and quantitative studies by also applying different types of open source software as and when necessary.

\section{Conflict of Interest}

The authors declare that there are no conflicts of interest in this paper.

\section{Source of Funding}

None.

\section{Reference}

1. Liner, B. M. (1998), "The NCSTRL approach to open architecture for the confederated digital library", D-Lib Magazine, available at; www.dlib.org/dlib/december98/liner/12leiner. Html

2. www.esmartlibraries.org.au/Pages/WhatIseSmartLibraries.asp $\mathrm{X}$

3. www.librarysolutions.com.au/Products.html

4. http://ebookfriendly.com/library-future-technologies/

5. www.edsurge.com > News > Postsecondary Learning > Higher Education

6. Pagore R. B.(2015) "Use of web 2.0 tools in libraries". In Role of Library and Librarian in college libraries $\left(1^{\text {st }}\right.$ ed. PP.111116), Aruna Publication.

7. www.canis.illinois.edu/news/Computerintro.pdf

8. Donald J. Water "What are Digital Libraries "council on Library and Information resources No:4 July/Aug 1998

9. www.australianscience.com.au/.../social-learning-impact-andcollaboration

10. www.carnegieuktrust.org.uk/CMSPages/GetFile.aspx? guid=25 0ba850...

11. https://en.wikipedia.org/wiki/Cloud_computing

12. https://en.wikipedia.org/wiki/Web_2.0

13. www.webology.org/2006/v3n2/a25.html

14. Pagore RB. Application of mobile Technology on Libraries. Int J Adv Innov Res. 2019;6/1(XVI), PP. 77-79.

15. Aswal, R. S. ed. (2003). Information Network in India. NewDelhi: Ess Ess Publication.

16. Dabas, C. "IT applications for TQM and Library marketing", New Delhi: Ess Ess Publications, 2008, p 40-42.

How to cite: Bhanudas RP. Relevance of it in libraries: Desire a tread a front to advanced library in current trends. IP Indian J Libr Sci Inf Technol. 2021;6(1):53-6. 